BREAKING THE HADRON STRING - QUARKS AT NAL?*

\author{
H. Goldberg \\ Department of Physicst \\ Northeastern University \\ Boston, Massachusetts 02115 \\ and \\ Stanford Linear Accelerator Center \\ Stanford University, Stanford, Calif. 94305
}

\begin{abstract}
We consider the average displacement from its near neighbors of a parton in a hadron string after absorbing a highly virtual photon. It is found that for $\mathrm{Q}^{2} \geq 60 \mathrm{GeV}^{2}$, the struck parton would find itself separated from its near neighbors by a distance greater than the average internucleon spacing in a nuclear matter. The consequences of this result vis a vis deep inelastic scattering of leptons from complex nuclei is discussed.
\end{abstract}

(Submitted to Phys。Rev。Lett。)

* Supported in part by the National Science Foundation, and in part by the U.S. Atomic Energy Commission.

$\dagger$ Permanent Address. 
The deep inelastic lepton-hadron scattering experiments at SLAC, ${ }^{1} \mathrm{CERN},{ }^{2}$ and NAL $^{3}$ seem to establish Bjorken scaling ${ }^{4}$ over a spectacular range of $Q^{2}$ and $\nu_{\circ}$ The simplest realization of Bjorken scaling is provided by the parton model, ${ }^{5}$ in which the currents scatter incoherently from elementary constituents within the nucleon. The proximity of the ratios $\sigma_{\nu} / \sigma_{\nu}$ and $-\int \mathrm{xF}_{3}(\mathrm{x}) \mathrm{dx} / \int \mathrm{F}_{2}(\mathrm{x}) \mathrm{dx}$ to the value $1 / 3$ and 1 , respectively, ${ }^{2}$ lend support to the characterization of partons as quarks. The validity of the impulse approximation requires that the forces responsible for binding the partons be fairly soft, ${ }^{5}$ and hence that a parton can travel freely for a considerable distance after being struck. The crisis is then clear: why do we not see quarks in nature? Various models of containment have been proposed, ${ }^{6}$ but the nature of their conclusions (i.e., absolute containment) makes an experimental test of these models extremely problematic。

In this note we present a discussion of containment based on the phenomenological dual string model of a hadron. ${ }^{7}$ We find that for $\mathrm{Q}^{2} \geq 60 \mathrm{GeV}^{2}$ (near $\omega \simeq 3$, the average displacement from its near neighbors of a parton struck by a virtual photon exceeds the average internucleon distance in a complex nucleus. It is then proposed that the normal string would fail to bring the struck parton back to the fold (because quarks of non-zero triality do scatter absorptively from nucleons - this we know from the existence of bilocal operators). We then discuss some possible experimental consequences of this result. We stress that in no way do we deal with the field theoretic basis of the String Hamiltonian - the latter is simply taken as a phenomenological description of the hadron dynamics .

\section{Breaking the String}

For simplicity, we work in an infinite momentum frame, and formulate the string model in the transverse $\mathrm{X}-\mathrm{Y}$ space. ${ }^{8}$ The partons are distributed along 
the $\theta$ coordinate with an average density ${ }^{8} \mathrm{dN} / \mathrm{d} \theta=(\pi \eta(\theta))^{-1}$, (where $\eta$ is the fractional longitudinal momentum) and the Hamiltonian which displaces the system in dilated time $\tau$ is ${ }^{8}$

$$
H=\frac{M^{2}}{2}=\frac{1}{2 \pi} \int_{0}^{\pi}\left[\left(\frac{\partial \vec{X}}{\partial \tau}\right)^{2}+G^{2}\left(\frac{\partial \vec{X}}{\partial \theta}\right)^{2}\right] d \theta+\text { const }
$$

The quantization is achieved through an expansion

$$
\underset{\sim}{X}(\tau)=\underline{x}_{c m}(\tau)+i \sum_{\ell}(\underbrace{a}(\ell) e^{-i G \ell \tau}-\underset{\sim}{a}(\ell) e^{+i G \ell \tau}) \frac{\cos \ell \theta}{\sqrt{\ell}}
$$

with the usual commutation relations $\left[a_{i}(\ell), a_{j}\left(\ell^{\prime}\right)\right]=\delta_{i j}{ }^{\delta} l^{\prime}$. The Hamiltonian can then be written as

$$
\mathrm{M}^{2}=2 \mathrm{G} \sum_{\ell} \ell \mathrm{n}(\ell)+\text { const }
$$

where $n(\ell)=\underline{a}^{+}(\ell) \cdot \underline{a}(\ell)$. It is easy to verify, for instance, that $(\partial \underline{X} / \partial \tau)=$ $\mathrm{i}[\mathrm{H}, \mathrm{X}(\tau)]$ 。 The function $\eta(\theta)$ is arbitrary, except that $\eta(0)=\eta(\pi)=0$ 。

We now propose the following physical picture at $\mathbf{P}=\infty$ : A parton with momentum $\eta \mathrm{P}$ in a hadron in its ground state is struck by a virtual photon with $\mathrm{q}_{\mu}=(\mathrm{m} \nu / \mathrm{P}, \mathrm{Q}, 0,0) .{ }^{9}$ The impulse approximation is valid in the IMF, since the time of interaction $t_{\text {int }} \sim\left(1 / q_{0}\right)_{I M F} \sim P / m v$, whereas typical lifetimes in the hadron are $\Delta \mathrm{t} \simeq 1 / \Delta \mathrm{E} \approx 2 \mathrm{P} / \Delta \mathrm{m}^{2}=\mathrm{P} / \mathrm{G}$, and $\mathrm{m} \nu>\mathrm{G}=1 / 2 \mathrm{GeV}{ }^{2}$. During the time of interaction the transverse momenta are $\ll$, and hence we have approximate energy-momentum conservation across the parton-photon vertex, ${ }^{9}$ with the resulting constraint $\omega \equiv 2 \mathrm{~m} \nu / \mathrm{Q}^{2}=\eta^{-1}$. Thus one fulfills the usual requisites of the parton model, and Bjorken scaling results. 
A long time after the interaction (at $\mathrm{t}>>\mathrm{P} / \mathrm{m} \nu$ but $\mathrm{t}<<\mathrm{P} / \mathrm{G}$ ) the struck parton will begin to interact with the remaining partons according to the string Hamiltoniam. The initial state (at $\tau=0$ in the IMF) will be taken to be

$$
\left.\left|Q, \theta>=e^{i Q X(\theta)}\right| 0\right\rangle
$$

where $10>$ is the target hadron ground state. This simply means that we translate the parton at position $\theta$ an amount $Q$ in the momentum space (we take $\left.\underline{Q}=Q \vec{\imath}_{\mathrm{X}}\right)$. The question now arises, what is the subsequent behavior of the partons in the string (especially of the struck parton)? In the case of a macroscopic chain of masses joined by springs, we know that a very strong impulse delivered to one of the masses will break the chain if the resulting separation from one of its neighbors is greater than some maximum value; the mass will probably be knocked out of the chain if its resulting displacement from the average position of its nearest neighbors becomes large. Let us try to apply these concepts to the hadronic string.

In calculating the mean square displacement of the struck parton from its nearest neighbors (henceforth called the strain), we must specify more carefully where the partons are located. In particular, from the density requiremont $\mathrm{dN} / \mathrm{d} \theta=[\pi \eta(\theta)]^{-1}$, we can see that the nearest neighbors of a parton $[\theta, \eta(\theta)]$ are located, on the average, at $\theta_{ \pm}=\theta \pm \pi \eta(\theta)$. A simple choice for $\eta(\theta)$ is $\eta(\theta)=\eta_{\max } \sin \theta,{ }^{8}$ and the lowest allowed value for $\eta_{\max }$ is $\pi^{-1}$ if the $\theta_{ \pm}$are to stay in the interval $[0, \pi]$ 。

This choice of $\eta(\theta)$ excludes consideration of the threshold region $\frac{1}{\pi}<\eta<1(1<\omega<\pi)$, and gives $\theta_{ \pm}=\theta \pm \sin \theta$. We are then interested in the 
displacement,

$$
\begin{aligned}
& -\Delta(\theta, \tau)=\underset{X}{X}(\theta, \tau)-\frac{1}{2}\left(\underline{X}_{+}\left(\theta_{+}, \tau\right)+\underset{X}{X}(\theta, \tau)\right) \\
& =i \sum_{\ell}\left(\underline{a}(\ell) e^{-i G \ell \tau}-\underline{a}^{+}(\ell) e^{i G \ell \tau}\right) \frac{2 \cos \ell \theta}{\sqrt{\ell}} \sin ^{2} \frac{1}{2} \ell \sin \theta
\end{aligned}
$$

Quantum mechanically, one computes

$$
\left\langle\Delta^{2}(\mathrm{Q}, \theta)\right\rangle=\left\langle\mathrm{Q}, \theta\left|\Delta^{2}(\theta, \tau)\right| \mathrm{Q}, \theta\right\rangle \text { averaged over } \tau
$$

This is easily done using the oscillator commutation relations, and the result is

$$
<\Delta^{2}\left(\mathrm{Q}^{2}, \theta\right)>=\mathrm{a}(\theta)+\frac{\mathrm{Q}^{2}}{\mathrm{G}} \mathrm{b}(\theta)
$$

where

$$
\begin{aligned}
& a(\theta)=\frac{8}{\mathrm{G}} \sum_{\ell=1}^{\ell} \frac{\cos ^{2} \ell \theta \sin ^{4}\left(\frac{1}{2} \ell \sin \theta\right)}{\ell} \\
& \mathrm{b}(\theta)=\frac{8}{\mathrm{G}} \sum_{\ell=1}^{\ell} \frac{\cos ^{4} \ell \theta \sin ^{4}\left(\frac{1}{2} \ell \sin \theta\right)}{\ell^{2}}
\end{aligned}
$$

As discussed in Ref. (8), the sums must be cut off for $\ell_{\max } \simeq E\left(\pi /\left(\theta_{i}-\theta_{i-1}\right)\right)$ $\simeq \mathrm{E}(\pi / \sin \theta)=\mathrm{E}\left(\eta^{-1}\right)=\mathrm{E}(\omega)$, where $\mathrm{E}(\mathrm{x})=$ greatest integer $\leq \mathrm{x}$ 。

In Fig. 1 we have plotted the strain $\left\langle\Delta^{2}>v s Q^{2}\right.$ for several values of $\omega=\pi / \sin \theta$. It is seen that for a given $Q^{2}$, a maximum strain is produced at the lowest value of $\omega$ available in our treatment $(\omega=\pi, \sin \theta=\pi / 2)$.

We now come to the crucial question: what is a reasonable upper bound to the strain, beyond which the hadron string fails to contain the parton? There is 
clearly no parameter in the string Hamiltonian itself which provides us with this scale. However, consider an experiment in which the leptons are scattered from complex nuclei. Once the strain exceeds about $(3 F)^{2}$, the struck parton would be screened from its nearest (parton) neighbors by a neighboring nucleon in the complex nucleus. It is a reasonable possibility that in this situation the trapping mechanism would fail (if the string does not break by this time anyhow). From Fig. 1, we see that $\left\langle\Delta^{2}\left(Q^{2}, \theta\right)\right\rangle=9 \mathrm{~F}^{2}$ when $\mathrm{Q}=55 \mathrm{GeV}^{2}$ at $\omega=3$. This region of $\left(Q^{2}, \omega\right)$ can be probed with both electron and neutrino beams at NAL。At higher values of $\omega$, much larger values of $Q^{2}$ are required. Are we committing ourselves to a prediction of quarks at NAL? We prefer to develop a more conservative approach to our result. We have found that a fairly reasonable phenomenological model of hadron dynamics becomes incapable of keeping a parton from being isolated from its near (parton) neighbors by nearby nucleons, when struck by a virtual photon of $\mathrm{Q}^{2} \geq 55 \mathrm{GeV}^{2}, \omega \simeq 3$, incident on a target of complex nuclei. What then happens? We consider three alternatives: (1) If no parton (quark) is seen, a simple explanation (on the phenomenological level) would be that the stiffness G (in Eq。(1)) is really a function of $\underset{X}{X}$ and that as $\underset{\sim}{X}$ becomes large, the stiffness increases so that the quark is contained to small displacements, no matter how hard it is struck. (2) A light quark is produced. (3) A quark is knocked out but once the "dressing" is complete, it is manifest as a very heavy particle $\left(\mathrm{M}^{2}>>1 \mathrm{GeV}^{2}\right)$. Even if the heavy quark is not observed, such an effect would destroy Bjorken scaling in the region of $Q^{2} \omega-M^{2}$. ${ }^{10}$ At any rate, we would encourage a careful study of the final state products of deep inelastic lepton-nucleus scattering in the region $\mathrm{Q}^{2} \geq 60 \mathrm{GeV}^{2}, \omega \simeq 3$.

Let us now deal with several questions which may have arisen in the reader's mind. 


\section{Lower Limit on $\Delta_{\max }^{2}$}

In the case where the quarks stay light when free, we can set a lower limit on the maximum strain, $\Delta_{\max }^{2}$. In such a case, a low-lying resonance (say $\mathrm{N}^{*}(1236)$ ) will undergo a spontaneous decay $\mathrm{N}^{*} \rightarrow$ qqq with reasonable probability unless $\left\langle\Delta^{2}(0, \theta)\right\rangle_{\text {res }} \approx\left\langle\Delta^{2}(0, \theta)\right\rangle_{\text {proton }} \equiv a(\theta)<\Delta_{\max }^{2}$ for all $\theta$. It turns out that the probability distribution $P\left(\Delta^{2}\right)$ is gaussian:

$$
\mathrm{P}\left(\Delta^{2}, \theta\right) \mathrm{d} \Delta^{2}=(\pi \mathrm{a}(\theta))^{-1} \mathrm{e}^{-\Delta^{2} / \mathrm{a}(\theta)} \mathrm{d} \Delta^{2}
$$

Also, numerically, $[\mathrm{a}(\theta)]_{\max } \simeq 0.25 \mathrm{~F}^{2}$. Experiments at $\mathrm{CERN}^{11}$ and Serpukhov ${ }^{11}$ have shown that quarks occur with a probability of less than $10^{-9}$ among charged secondaries in pp collisions. With 6-7 charged secondaries/ event, and perhaps 2 resonances/event, one obtains an upper limit of $10^{-9}\left(e^{-20.7}\right)$ for the branching ratio resonance $\rightarrow$ quarks. This is achieved with $\Delta_{\max }^{2}>[\mathrm{a}(\theta)]_{\max }(20.7)=5.1 \mathrm{~F}^{2}$.

\section{Fluctuations}

The value of $Q^{2}$ needed to produce an average strain $\left\langle\Delta^{2}(\theta)\right\rangle$ is $\mathrm{Q}_{0}^{2} \sim \mathrm{G}<\Delta^{2}>/ \mathrm{b}(\theta)(\mathrm{Eq} \cdot(8))$. One can show that the probability of producing such a strain with a lower value of $Q^{2}$ is negligible unless $Q^{2} \geq 0.9 Q_{0}^{2}$.

\section{Recombination with Other Partons}

We can also show that the mean square separation of the struck parton from any other parton in the string is larger than the strain $\Delta^{2}$. So recombination of the struck parton with others in the string is unlikely. 
Strains in Purely Hadronic Interactions

It is very difficult to produce a large strain by exciting the string through purely hadronic interactions at presently available energies. To see this, we calculate the average strain at position $\theta$ in the string for a highly excited hadron $\left\{\{n(l), \ell\}>\right.$, where $M^{2}=2 G \Sigma \ell n(\ell)$. The result is

$$
\left\langle\Delta^{2}\left(M^{2}, \theta\right)\right\rangle=\frac{8}{\mathrm{G}} \sum_{\ell=1}^{\omega=\pi / \sin \theta}(\mathrm{n}(\ell)+1) \frac{\cos ^{2} \ell \theta \sin ^{4}\left(\frac{1}{2} \ell \sin \theta\right)}{\ell}
$$

What is the most probable distribution $n(\ell)$ at a given mass $M^{2}$ ? We take for this the Bose form ${ }^{12}$

$$
n(\ell)=\bar{n}(\ell)=\frac{1}{e^{\ell / t}-1}
$$

which satisfies $2 G \Sigma \ell n(\ell)=M^{2}$ for $t=(\sqrt{3} / \pi) M / \sqrt{G}$, and leads to a correct counting of the degeneracy of levels in the dual resonance model ${ }^{13}$ The resulting

$$
\left\langle\Delta^{2}\left(\mathrm{M}^{2}, \theta\right)\right\rangle=\frac{8}{\mathrm{G}} \sum_{\ell=1}^{\pi / \sin \theta} \frac{1}{1-\mathrm{e}^{-\ell / \mathrm{t}}} \frac{\cos ^{2} \ell \theta \sin ^{4} \frac{1}{2} \ell \sin \theta}{\ell}
$$

will be small unless (1) $\ell<t$ for some $\ell$, and (2) $\ell \sim \ell_{\max }-\pi / \sin \theta$ for the same $\ell$ (so that $\sin ^{4} \frac{1}{2} \ell \sin \theta$ should not be $\ll 1$ ). This will only occur if $\ell \ll t$ for every $\ell \leq \ell$ max ${ }^{\circ}$

The statistical factor $n(l)+1$ then becomes $\simeq t / \ell$ over the whole range of $\ell$ in the summation, and we have the result

$$
\begin{aligned}
<\Delta^{2}\left(M^{2}, \theta\right)> & =\frac{8 t}{G} \sum_{\ell=1}^{\omega} \frac{\cos ^{2} \ell \theta \sin ^{4} \frac{1}{2} \ell \sin \theta}{\ell} \\
& =t b(\theta)
\end{aligned}
$$


Compare Eqs。(13), (6) and (8), we find that to produce the strain equivalent to that attained with a given value of $\mathrm{Q}^{2}$, one must have

$$
\mathrm{t} \simeq \mathrm{Q}^{2} / \mathrm{G}
$$

or

$$
\mathrm{M}=\frac{\pi}{\sqrt{3}} \frac{\mathrm{Q}^{2}}{\sqrt{\mathrm{G}}}
$$

We have estimated that $Q^{2}>40 \mathrm{GeV}^{2}$ may be necessary to break the string. Via Eq. (14), the excitation of a fireball with a mass $>80 \mathrm{GeV} / \mathrm{c}^{2}$ is required to produce the same strain. This is out of range of present accelerators. For instance, to diffractively produce this mass at $\mathrm{x} \simeq 0.9$ in $\mathrm{pp} \rightarrow \mathrm{pX}$, we would need an $\mathrm{s}=\frac{\mathrm{M}^{2}}{1-\mathrm{x}}=10 \mathrm{M}^{2}>64,000 \mathrm{GeV}^{2}$. This requires colliding beams of $130 \mathrm{GeV}$ protons or cosmic rays with $\mathrm{E}>30 \mathrm{TeV}$.

If the spectrum of hadron states excited in $\mathrm{e}^{+} \mathrm{e}^{-}$collisions is also characterized by $\bar{n}(\ell)$, then in that process as well the strains would be small at least until $\mathrm{E}_{+}+\mathrm{E}_{-} \doteq 100 \mathrm{GeV}$.

$\underline{\text { Conclusions }}$

The large level spacing implied by the dual string sets a very small length scale $\left(-(2 \mathrm{G})^{-1}=0.04 \mathrm{~F}^{2}\right)$. Thus high $\mathrm{Q}^{2}$ values are tolerated before displacements in the string exceed $1 \mathrm{~F}$ - no now dynamics (beyond the unknown field theory which underlies the string Hamiltonian) nced be invoked to contain quarks until $\mathrm{Q}^{2} \approx 25 \mathrm{GeV}$. Around $\mathrm{Q}^{2} \approx 60 \mathrm{GeV}^{2}$ (with $\omega \simeq 3$ ), the strains become larger than the internuclear distances in a complex nucleus, and the string trapping mechanism may fail to gather partons back into the nucleon if the experiment is done with a complex nucleus target. ${ }^{14}$ Whether at this point light quarks are observed, heavy quarks are produced and scaling is destroyed, 
or nothing happens (indicating that partons are truly a mathematical fiction underlying the light-cone algebra) remains to be seen in the new generation of lepton-hadron experiments.

I would like to thank Drs. J.D.Bjorken, R. Blankenbecler, S. J. Brodsky and M. -S. Chen for some helpful conversations.

\section{REFERENCES}

1. G. Miller, et al。, Phys. Rev. D $\underline{5}, 528$ (1972).

2. T. Eichten, et al., Phys. Letters $\underline{46}$ B, 274 (1973).

3. B. Barish, talk presented at Irvine Conference on Lepton-Hadron Interactions (Dec。1973).

4. J.D. Bjorken, Phys. Rev。 179, 1547 (1969).

5. R.P.Feynman, Phys. Rev. Letters 23, 1415 (1969); J.D。Bjorken and E.Paschos, Phys。Rev. 185, 1975 (1969)。

6. K. Johnson, Phys。Rev. D 6 , 1101 (1972); A. Casher, J. Kogut and L. Susskind, Phys. Rev. Letters $\underline{31}, 972$ (1973); A. Chodos, R。L. Jaffe, K. Johnson, C.B. Thorn and V.F. Weisskopf, MIT preprint "A New Extended Model of Hadrons," C.T.P. publication No. 387 (unpublished)。

7. See for instance, $Y$. Nambu, "Quark Model and the Factorization of the Veneziano Amplitude," talk presented at the International Conference on Symmetries and Quark Models, Wayne State University, 1969; L。Susskind, Phys。Rev。D $1,182(1970)$.

8. J. Kogut and L. Susskind, Physics Reports 8 C, 73 (1973)。

9. S.D. Drell and T.M. Yan, Ann. Phys。(N.Y.) 66, 378 (1971)。

10. For instance, the energy-momentum conservation condition at the photonvirtual parton-real parton vertex introduces a factor

$$
\begin{gathered}
\delta\left((\mathrm{p}+\mathrm{q})^{2}-\mathrm{M}^{2}\right) \propto \delta\left(\eta-\frac{\mathrm{Q}^{2}+\mathrm{M}^{2}}{2 \mathrm{~m} \nu}\right) . \\
-10-
\end{gathered}
$$




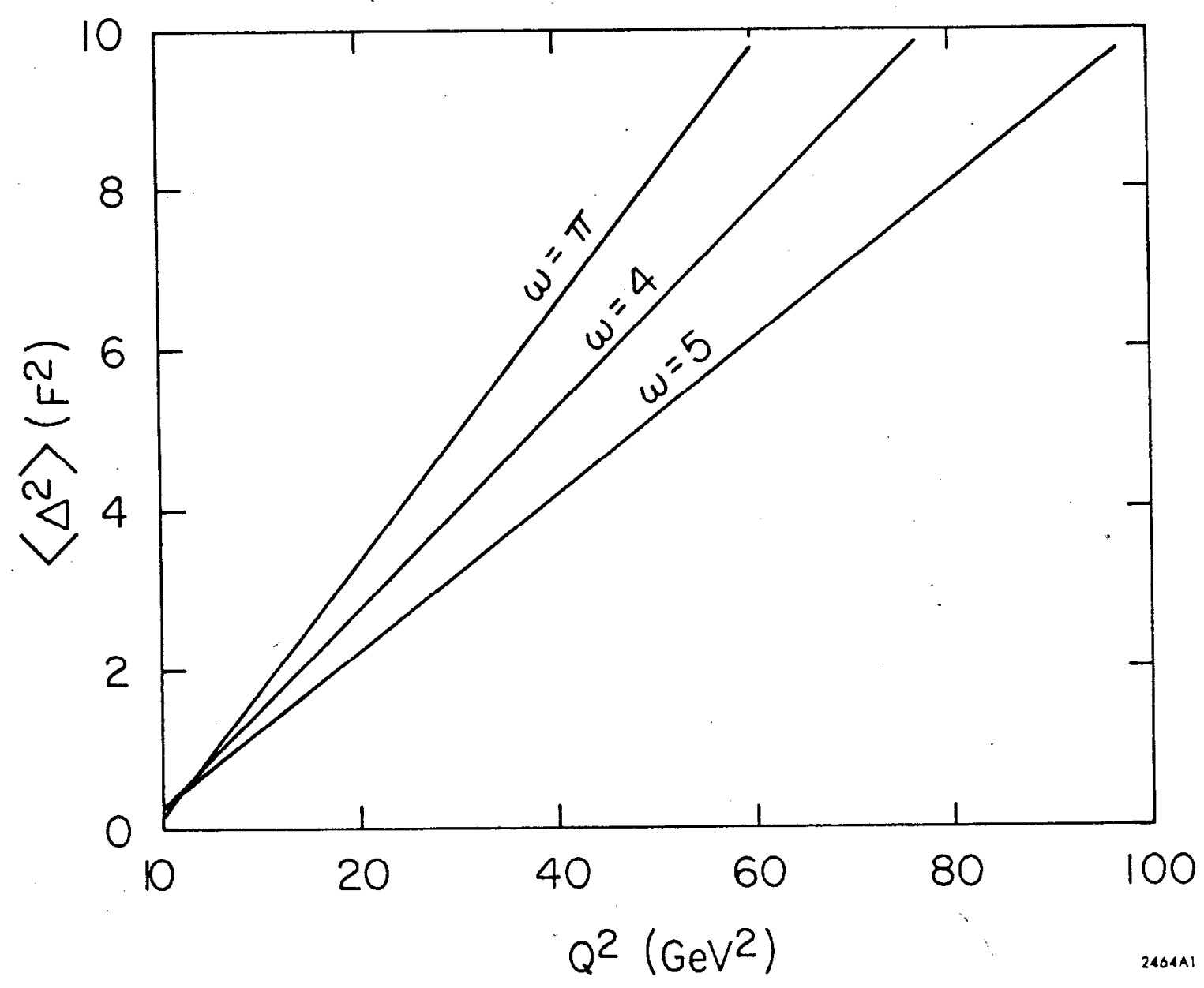

